\title{
Eccentric connectivity index of unicyclic graphs with application to cycloalkanes
}

\begin{abstract}
Let $\mathrm{G}$ be a simple connected molecular graph. The eccentric connectivity index $3(\mathrm{G})$ is defined as $\boldsymbol{z}^{r}(\mathrm{G})=\times 3 \in \mathrm{V}(\mathrm{G}) \operatorname{deg}(3) \mathrm{ec}(3)$, where $\operatorname{deg}(3)$ denotes the degree of vertex $\mathrm{v}$ and ec(3) is the largest distance between 3 and any other vertex $u$ of G. In this paper, we construct the general formulas for the eccentric connectivity index of unicyclic graphs with application to cycloalkanes.
\end{abstract}

Keyword: Cycloalkanes; Eccentric connectivity index; Unicyclic chemical graphs; Unicyclic graphs 